\title{
Estimativa de parâmetros genéticos, componentes de (co)variância e tendências genéticas e fenotípicas para características produtivas pré- desmama em bovinos Devon no Rio Grande do Sul ${ }^{1}$
}

\author{
Marcela Bicca Bragança Corrêa ${ }^{2}$, Nelson José Laurino Dionello ${ }^{3}$, Fernando Flores Cardoso 4 \\ ${ }^{1}$ Parte da dissertação apresentada pela primeira autora à FAEM/UFPEL para obtenção do título de Mestre em Ciências. Financiada pela \\ CAPES. \\ 2 Mestranda do Departamento de Zootecnia/FAEM/UFPEL.Bolsista CAPES \\ ${ }^{3}$ Departamento de Zootecnia/FAEM/UFPEL. \\ ${ }^{4}$ Embrapa Pecuária Sul.
}

RESUMO - Os objetivos neste estudo foram estimar componentes de (co)variância e parâmetros genéticos, como herdabilidades e correlações, e verificar as tendências genéticas e fenotípicas para os pesos ao nascer (PN) e à desmama (PD) e o ganho do nascimento à desmama ajustado para 205 dias (GNDA). Foram utilizados registros de 23.369 animais da raça Devon, coletados pelo PROMEBO - Programa de Melhoramento de Bovinos de Carne, conduzido pela Associação Nacional de Criadores - "Herd Book Collares", nascidos entre 1980 e 2000. Desses animais, 16.593 tinham dados de PN, 22.530 tinham registros de PD e 22.259 possuíam dados de GNDA. As análises foram realizadas pelo método da máxima verossimilhança restrita, pelo programa MTDFREML. Inicialmente, foram realizadas análises univariadas de cada característica e, posteriormente, análises bivariadas relacionando os pesos, tanto ao nascer quanto à desmama, ao ganho de peso pré-desmama. As herdabilidades e os erros-padrão obtidos das análises univariadas foram de $0,32 \pm 0,03$ para PN, 0,17 $\pm 0,01$ para GNDA e de $0,12 \pm 0,01$ para PD. Nas análises bivariadas do PN com GNDA e do PD com GNDA, as herdabilidades obtidas foram de 0,35 e 0,17 e de 0,32 e 0,42 , respectivamente. Os resultados demonstram, pelas herdabilidades médias para os pesos e ganhos de peso, que essas características podem responder de forma eficiente à seleção. As correlações genéticas, alta $(0,95)$ entre PD e GNDA e baixa $(0,15)$ entre PN e GNDA, demonstram que, ao selecionar pelo ganho, é possível obter uma resposta correlacionada no peso à desmama sem acarretar incremento no PN. As tendências genéticas e fenotípicas obtidas demonstram a ocorrência de progresso genético para as características de GNDA e PD, enquanto PN apresenta-se em platô, indicando, portanto, mudança favorável no desempenho pré-desmama, sem aumento no risco de problemas de parto.

Palavras-chave: correlações, efeito materno, herdabilidade, pré-desmama

\section{Estimation of genetic parameters and (co) variance components for pre- weaning productive traits in Devon Cattle in Rio Grande do Sul}

\begin{abstract}
The objective of this study was to estimate (co)variance components and genetic parameters, such as heritabilities and correlations, and genetic and phenotypic trends for birth weight (PN), weaning weight (PD) and 205 day adjusted weight gain (GNDA). A total of 23,369 records on animals, born from 1980 through 2000, collected by PROMEBO - beef cattle improvement program of Associação Nacional de Criadores Herd Book Collares, were used. Of these animals, 16,593 had record of PN, 22,530 had of PD and 22,259 of GNDA. Analyses were performed by the restricted maximum likelihood method using the MTDFREML program. Initially, a univariate analysis was used for each trait and, afterwards, bivariate models were used in analyses relating weights at birth and weaning with pre-weaning gain Heritabilities and standard errors obtained from univariate analyses were of $0.32 \pm 0.03$ for PN, $0.17 \pm 0.01$ for GNDA and of $0,12 \pm 0,01$ for PD. From bivariate analyses of PN with GNDA and of PD with GNDA, heritability estimates were of 0.35 and 0.17 and of 0,32 and 0.42 , respectively. Medium heritabilities for weights and weight gains indicate that these traits efficiently respond to selection. Genetic correlation estimates was high (0.95) between PD and GNDA and low (0.15) between PN and GNDA indicating that selection for weight gain make it possible to obtain a correlated response on weaning weight, without increasing birth weight. Estimated genetic and phenotypic trends demonstrated genetic progress for GNDA and PD, while PN is on plateau, thereby, indicating favorable change in pre-weaning performance, without increasing risk of calving difficulty.
\end{abstract}

Key Words: correlations, heritability, maternal effect, pre-weaning 


\section{Introdução}

A intensa busca por maior produtividade nos últimos anos no intuito de transformar a bovinocultura de corte em uma atividade de maior rentabilidade trouxe para os produtores uma preocupação constante com a genética dos rebanhos. Isto ocorre porque sabe-se que uma das alternativas para modificar a eficiência dos sistemas de produção é a utilização de animais geneticamente superiores para características de importância econômica. Segundo Fernandes et al. (2002), a resposta a determinado manejo nutricional é diretamente proporcional à superioridade genética dos animais utilizados na propriedade.

É de grande importância quando se pretende uma seleção genética eficiente a estimativa das herdabilidades direta e materna da característica estudada na população, uma das principais ferramentas do melhoramento animal (Biffani et al., 1999), visto que características com estimativas de baixa herdabilidade não apresentam respostas satisfatórias ao trabalho de seleção dos animais (Fernandes et al., 2002). Portanto, os métodos de seleção e o progresso genético dependem do conhecimento dos parâmetros genéticos (herdabilidades e correlações genéticas) das populações.

Uma estratégia para se avaliar o progresso genético do rebanho é por meio da tendência genética da população, para características de importância econômica ao longo dos anos, o que é possível pelo cálculo das DEPs (diferença esperada da progênie) dos animais. Juntamente com a tendência genética, é desejável que se avalie a tendência fenotípica deste mesmo rebanho para analisar se o progresso fenotípico ocorre em decorrência da genética dos indivíduos ou se o programa de seleção deve sofrer modificação nos critérios seletivos.

Mudanças morfofisiológicas ao longo da vida do animal são responsáveis pela variação dos pesos dos seus produtos (Martins et al., 2000).

O efeito materno sobre o desenvolvimento do bezerro, expresso pelo ganho de peso, varia segundo as diferenças permanentes entre vacas, nos período de gestação e aleitamento, do ambiente uterino, da produção de leite e da habilidade materna e, inclusive, da imunidade passiva obtida pela ingestão de colostro (Koch, 1972).

O efeito da idade da vaca é importante porque o desempenho dos bezerros melhora com o aumento da idade da vaca, atingindo o pico, geralmente aos sete anos (maturidade fisiológica da habilidade materna e pico de produção de leite). Antes ou a partir desta idade, o desempenho é menor (Dal-Farra et al., 2002), demonstrando que, geralmente, vacas jovens, por não terem ainda completado seu desenvolvimento, e vacas velhas desmamam bezerros mais leves que as vacas maduras.
O crescimento do bezerro é fator determinante da produtividade dos sistemas de produção de carne. O peso ao nascer e o ganho até a desmama são os componentes iniciais do crescimento e são determinados pelo potencial genético e pelo ambiente no qual os animais são criados. Estas características apresentam herdabilidades de médio a altas (20 a 40\%) (Cardellino \& Osório, 1998).

O ambiente materno é de grande importância, pois, nos três primeiros meses de vida, o crescimento do bezerro depende da produção de leite da mãe (Koch, 1972).

Segundo Martins et al. (2000), os pesos ao nascer e à desmama (205 dias) e o ganho de peso do nascimento à desmama refletem, de modo geral, a habilidade materna da vaca e a capacidade do animal em se desenvolver. O peso ao nascer possibilita o ajuste dos dados às idades padrões e ao peso à desmama. Segundo Lobo (1994), possibilita ainda a avaliação genética de matrizes pelo índice de fertilidade real ( $\mathrm{kg}$ de bezerro desmamado/vaca/ano).

Há uma marcada diferença nestes parâmetros entre populações criadas em ambientes diferentes (Koots et al., 1994). Estimativas confiáveis de parâmetros genéticos das populações locais são necessários à adequada condução dos programas de melhoramento genético em cada região. Alguns autores têm utilizado modelos animais para estimativas dos efeitos genéticos direto e materno em bovinos Nelore (Eler et al., 1995; Mercadante \& Lobo,1997) e em bovinos Aberdeen Angus (Cardoso, 1999). A literatura nacional, contudo, ainda carece de estudos de parâmetros genéticos em bovinos da raça Devon, considerando efeitos genéticos direto e materno.

O objetivo neste estudo foi estimar os parâmetros genéticos (herdabilidades e correlações), os componentes de (co)variância e as tendências genéticas e fenotípicas para os pesos ao nascer e à desmama e o ganho de peso pré-desmama.

\section{Material e Métodos}

O estudo foi desenvolvido utilizando-se dados coletados rotineiramente pelo PROMEBO - Programa de Melhoramento de Bovinos de Carne, conduzido pela Associação Nacional de Criadores - "Herd Book Collares", de animais da raça Devon que participaram do programa entre os anos de 1980 e 2000. Foram utilizados 23.369 registros, incluindo 16.593 de peso ao nascer (PN), 22.530 de peso à desmama (PD) e 22.259 para o ganho de peso do nascimento à desmama ajustado para 205 dias (GNDA).

O PN foi obtido pela pesagem nas primeiras 24 horas de vida do animal, no momento das anotações do nascimento, com balança a campo. $\mathrm{O}$ peso à desmama $\mathrm{PD}$ foi obtido no momento da desmama dos animais, com jejum prévio de 12 
horas, com os animais separados das mães, para evitar distorções em decorrência da ingestão de líquidos. A partir dos dois pesos, foi obtido o GNDA, a partir da equação: GNDA $=((\mathrm{PD}-\mathrm{PN}) / \mathrm{IT}) \times 205$, em que IT é a idade do bezerro, em dias, no momento da desmama.

Todas as informações disponíveis de genealogia foram utilizadas, principalmente de touros, por meio de pesquisa nos registros do "Herd Book Collares".

Foi utilizado o seguinte modelo estatístico:

$$
\mathrm{y}=\mu+\mathrm{X} \beta+\mathrm{Zg}+\mathrm{Mm}+\mathrm{Wp}+\mathrm{e}
$$

em que: $\mathrm{y}=$ vetor de observações; $\mu=$ vetor de médias; $\beta=$ vetor de efeitos fixos (grupo de contemporâneos; data juliana de nascimento; idade da vaca; idade do bezerro); $\mathrm{X}=$ matriz de incidência dos efeitos fixos; $\mathrm{g}=$ vetor dos efeitos genéticos aditivos diretos; $Z=$ matriz de incidência dos efeitos genéticos aditivos diretos; $m=$ vetor de efeitos genéticos aditivos maternos; $\mathrm{M}=$ matriz de incidência dos efeitos genéticos aditivos maternos; $p=$ vetor dos efeitos de ambiente materno permanente; $\mathrm{W}=$ matriz de incidência dos efeitos de ambiente materno permanente; e corresponde ao erro residual.

Para o modelo, são válidas as seguintes condições:

$$
\mathrm{E}[\mathrm{y}]=\mu+\mathrm{X} \beta
$$

$$
E\left[\begin{array}{c}
g \\
m \\
p \\
e
\end{array}\right]=\left[\begin{array}{l}
0 \\
0 \\
0 \\
0
\end{array}\right] \operatorname{Var}\left[\begin{array}{c}
g \\
m \\
p \\
e
\end{array}\right]=\left[\begin{array}{cccc}
A \sigma_{g}^{2} & A \sigma_{g m} & 0 & 0 \\
A \sigma_{g m} & A \sigma_{m}^{2} & 0 & 0 \\
0 & 0 & I_{n c} \sigma_{p}^{2} & 0 \\
0 & 0 & 0 & I_{n} \sigma_{e}^{2}
\end{array}\right]
$$

em que nc é o número de vacas e n, o número de observações; A, a matriz de parentesco e I, as matrizes identidade; $\sigma^{2}$, a variância genética aditiva direta; $\sigma_{m}^{2}$, a variância genética aditiva materna; $\sigma_{\mathrm{gm}}$, a covariância entre os efeitos aditivos direto e materno; $\sigma_{c}^{2}$, a variância de ambiente permanente da vaca; e $\sigma_{\text {e }}^{2}$, a variância ambiental.

Da mesma forma que em Cardoso et al. (2001), o termo $\mathrm{Mm}$ do modelo geral (efeito genético materno) não foi incluído na estimação dos parâmetros genéticos para PN. O termo $\mathrm{Wp}$ do modelo geral foi mantido com a finalidade de considerar os efeitos maternos permanentes sobre esta característica.

Os grupos de contemporâneos foram formados incluindo criador-rebanho, ano da produção (safra), estação de produção, sexo, grupo de manejo (alimentar) e data da pesagem de desmama. Grupo de contemporâneos com apenas um animal e touros com somente um filho foram previamente excluídos da análise, pois não fornecem informações relevantes para comparações.

As estimativas para efeitos fixos (grupo de contemporâneos e covariáveis) e efeitos aleatórios foram obtidas pela metodologia dos modelos mistos, utilizando-se um modelo animal, e os componentes de (co)variância foram estimados por máxima verossimilhança restrita, utilizando-se o programa MTDFREML (Boldman et al., 1995).

Inicialmente, foram realizadas análises univariadas das características avaliadas, que, posteriormente, serviram de base comparativa para as análises bivariadas do GNDA com PN e GNDA com PD. Nestas análises, o efeito materno e não-correlacionado de ambiente permanente da vaca foi considerado apenas para o GNDA.

A herdabilidade para mérito genético total (direto + materno) na característica GNDA foi calculada como proposto por Willham (1972):

$$
\mathrm{h}_{\mathrm{t}}^{2}=\left(\sigma_{\mathrm{g}}^{2}+0,5 \sigma_{\mathrm{m}}^{2}+1,5 \sigma_{\mathrm{gm}}\right) / \sigma_{\mathrm{f}}^{2}
$$

em que $\sigma_{f}^{2}$ é a variância fenotípica, determinada pela seguinte expressão:

$$
\sigma_{\mathrm{f}}^{2}=\sigma_{\mathrm{g}}^{2}+\sigma_{\mathrm{m}}^{2}+\sigma_{\mathrm{gm}}+\sigma_{\mathrm{c}}^{2}+\sigma_{\mathrm{e}}^{2}
$$

Para o cálculo das tendências genéticas, foram necessárias análises univariadas das características, as DEPs diretas e maternas de cada indivíduo. Posteriormente, os animais foram separados por ano de nascimento para possibilitar o cálculo das DEPs anuais médias para o período de 1980 a 2000. A partir dos valores das DEPs anuais médias, calculou-se sua regressão em relação ao ano de nascimento dos animais, utilizando-se o PROC REG do SAS (1996), seguindo tendência linear. Para efeitos comparativos, foi também obtida a tendência fenotípica da população, pela regressão dos valores médios obtidos para as características, pelo ano de nascimento do indivíduo, pelo mesmo modelo de análise das DEPs. Assim, verificaram-se as evoluções genética e fenotípica simultaneamente, ou não.

\section{Resultados e Discussão}

Os componentes de covariância e os parâmetros genéticos para as características estudadas, resultantes de análises univariadas, são apresentados na Tabela 1.

A herdabilidade para o PN foi de 0,32 ou $32 \%$, o que está de acordo com os resultados encontrados na literatura, como os reportados por Boligon et al. (2004), que obtiveram herdabilidade de $0,33 \mathrm{em}$ animais da raça Charolês no Rio Grande do Sul, valor semelhante ao encontrado por Laureano et al. (2004), que obtiveram 0,30 em bovinos Nelore, e superior ao obtido por Cardoso (1999) e Silva et al. (2004), de 0,29 e 0,13, respectivamente, para animais Angus e Guzerá. Martins et al. (2000) encontraram herdabilidade bastante alta, de 0,59 para PN de bovinos Nelore no Maranhão, corroborando os resultados descritos por Fernandes et al. (2002), que obtiveram herdabilidade de 0,54 para PN do rebanho Charolês no Rio Grande do Sul. 
Tabela 1 - Estimativas dos componentes de variância e parâmetros genéticos com erros-padrão para o peso ao nascer $(\mathrm{PN})$, o ganho de peso do nascimento à desmama ajustado para 205 dias (GNDA) e o peso à desmama (PD), a partir de análises univariadas

Table 1 - Estimates of variance components, genetic parameters and respective standard errors for birth weight (BW), 205 day adjusted weaning gain (WG) and weaning weight (WW), from univariate analyses

\begin{tabular}{lccc}
\hline Característica & PN & GNDA & PD \\
Trait & $B W$ & $W G$ & $W W$ \\
\hline
\end{tabular}

Componentes de variância Variance components

$\begin{array}{ll}\sigma^{2}{ }^{\mathrm{a}} & 1,55 \\ \sigma_{\mathrm{m}}^{2} & \\ \sigma_{\mathrm{gm}} & \\ \sigma^{2} \mathrm{c} & 0,23 \\ \sigma^{2} & 2,84 \\ \sigma_{\mathrm{f}}^{2} & 4,50\end{array}$

$\begin{array}{cc}88,48 & 54,14 \\ 64,41 & 49,68 \\ -38,97 & -15,11 \\ 72,54 & 67,34 \\ 330,78 & 306,55 \\ 517,24 & 462,62\end{array}$

Parâmetros genéticos Genetic parameters

$\begin{array}{cccc}\mathrm{h}^{2}{ }^{\mathrm{a}} & 0,32 \pm 0,03 & 0,17 \pm 0,01 & 0,12 \pm 0,01 \\ \mathrm{~h}^{2}{ }_{\mathrm{m}} & & 0,12 \pm 0,01 & 0,11 \pm 0,01 \\ \mathrm{r}_{\mathrm{gm}} & & -0,52 \pm 0,01 & -0,29 \pm 0,01 \\ \mathrm{c}^{2} & 0,05 \pm 0,01 & 0,14 \pm 0,01 & 0,15 \pm 0,01 \\ \mathrm{e}^{2} & 0,63 \pm 0,04 & 0,64 \pm 0,01 & 0,66 \pm 0,03 \\ \mathrm{~h}^{2} & & 0,12 & 0,12\end{array}$

$\frac{\mathrm{h}_{\mathrm{t}}{ }_{\mathrm{t}}}{\sigma^{2}{ }^{2}=\text { Variância genética aditiva direta, } \sigma^{2}{ }_{\mathrm{m}}=\text { variância genética aditiva }}$ materna, $\sigma_{\mathrm{gm}}=$ covariância genética entre os efeitos aditivos direto e materno, $\sigma_{c}^{2}=$ variância de ambiente permanente, $\sigma^{2}{ }_{\mathrm{e}}=$ variância ambiental, $\sigma^{2}{ }_{f}=$ variância fenotípica, $h^{2}{ }_{a}=$ herdabilidade genética aditiva, $h^{2}{ }_{m}$ herdabilidade aditiva materna, $r_{\mathrm{gm}}=$ correlação genética entre efeitos aditivos direto e materno, $c^{2}=$ porção da variância devido ao ambiente permanente, $\mathrm{e}^{2}=$ porção da variância devido ao ambiente temporário e $\mathrm{h}^{2}{ }_{\mathrm{t}}=$ herdabilidade total.

$\sigma_{a}^{2}=$ direct additive genetic variance, $\sigma_{m}^{2}=$ maternal additive genetic variance, $\sigma_{g m}=$ genetic covariance between the direct additive effect and maternal additive effect, $\sigma^{2}=$ permanent environmental variance, $\sigma_{e}^{2}=$ temporary environmental variance, $\sigma_{f}^{2}=$ phenotypic variance, $h^{2}{ }_{a}=$ direct additive genetic heritability, $h^{2}{ }_{m}=$ maternal additive genetic heritability, $r_{g m}=$ genetic correlation between the direct additive effect and maternal additive effect, $c^{2}=$ permanent environmental variance, $e^{2}=$ temporary environmental variance and $h^{2}{ }_{t}=$ total heritability.

Estes resultados indicam que, mesmo havendo diferenças relevantes entre as estimativas, a magnitude de herdabilidade para esta característica varia de média à alta; ou seja, há grande participação da variação genética aditiva na transmissão desta característica e sua utilização na seleção é eficiente para o progresso genético do rebanho.

Para GNDA, a herdabilidade encontrada foi de 0,17, inferior às relatadas por Cardoso (1999), de 0,25, para bovinos Aberdeen Angus no Rio Grande do Sul, e por Laureano et al. (2004), para bovinos da raça Nelore $(0,23)$. Porém, foi superior à encontrada por Fernandes et al. (2002), de 0,12 , para a raça Charolês no Rio Grande do Sul. O efeito materno é de grande importância no desempenho do indivíduo nesta fase do crescimento; tanto que a herdabilidade materna $(0,12)$ foi superior à encontrada por Fernandes et al. (2002), de 0,05. Laureano et al. (2004) obtiveram 0,09 para animais da raça Nelore e Boligon et al. (2004) encontraram 0,02, também para animais do rebanho Charolês no Rio
Grande do Sul, expostos ao mesmo clima que o rebanho deste estudo. O efeito materno total para GNDA foi de 0,26 ( 0,12 da herdabilidade materna $+0,14$ do ambiente permanente da vaca) e está de acordo com o reportado por Laureano et al. (2004), que obtiveram efeito materno total para GNDA de $0,23(0,10+0,13)$.

Para PD, a herdabilidade de 0,12 está bem abaixo das descritas por Martins et al. (2000), de 0,42, Laureano et al. (2004), de 0,31, e Boligon et al. (2004), de 0,21. Porém, está próxima da estimativa relatada por Ferraz Filho et al. (2002), de 0,16 . Lima et al. (2004) obtiveram herdabilidade de $0,17 \mathrm{e}$ Silva et al. (2004), de 0,10. Da mesma forma que para as demais características, o efeito materno novamente mostrou-se de grande importância. Sua inclusão no modelo denota confiabilidade nas estimativas, pois, pelos resultados obtidos para PD, uma herdabilidade materna de $0,11 \mathrm{e}$ 0,15 como a porção da variância decorrente do ambiente permanente da vaca é bastante considerável, corroborando os achados de Ferraz Filho et al. (2002), que estimaram herdabilidade materna de 0,10, Lima et al. (2004), de 0,14, e Laureano et al. (2004), de 0,09.

A herdabilidade aditiva materna médio-baixa para o GNDA e PD indica que é possível aumentar o desempenho para o ganho de peso pré-desmama pela seleção das fêmeas com maior habilidade materna. Entretanto, para aumentar a precisão de seleção, deve-se considerar na avaliação individual o desempenho de parentes por meio dos laços genéticos. Houve antagonismo entre os efeitos genéticos direto e materno para as características $\left(\mathrm{GNDA}_{\mathrm{gm}}=-0,52\right.$ e $\mathrm{PD}_{\mathrm{gm}}=-0,29$ ).

Os resultados obtidos para as covariâncias e correlações genéticas das análises bivariadas entre GNDA/PN e GNDA/PD são apresentados na Tabela 2.

A correlação genética aditiva direta entre GNDA/PN de 0,15 foi inferior à encontrada por Laureano et al. (2004), de 0,55 para bovinos Nelore, e Lobo et al. (2000), de 0,42. Porém, é muito inferior à relatada por Martins et al. (2000), de 0,77. Cardoso et al. (2001) encontraram correlação entre PN e GNDA de -0,06, o que, segundo os autores, demonstra independência genética entre as características e que a seleção para maior ganho de peso do nascimento à desmama não implica necessariamente em incremento de PN. A estimativa de correlação de 0,15 neste estudo permite inferir que, por apresentar baixa magnitude, as características têm apenas pequena proporção determinada pelos mesmos genes. A correlação GNDA/PD de 0,95 foi bem superior, corroborando a obtida por Martins et al. (2000), de 0,83. O resultado das correlações genéticas diretas do GNDA/PD demonstram que a seleção pelo ganho de peso ou pelo peso à desmama pode implicar resposta correlacionada para 
outra característica, sem causar incremento significativo no PN, o que seria desfavorável pela possibilidade de causar problemas de parto.

As herdabilidades obtidas na análise bivariada entre o PN e GNDA foram de 0,35 e 0,17, respectivamente. Os

Tabela 2 - Covariâncias (acima da diagonal) e correlações (abaixo da diagonal) para peso ao nascer (PN), ganho de peso do nascimento à desmama ajustado para 205 dias (GNDA) e peso à desmama (PD), a partir de análises bivariadas

Table 2 - Covariances (above diagonal) and correlations (below diagonal) for birth weight (BW), 205 day adjusted weaning gain (WG) and weaning weight (WW), from bivariate analyses

\begin{tabular}{lccc}
\hline Característica & PN & GNDA & PD \\
Trait & $B W$ & $W G$ & $W W$ \\
\hline
\end{tabular}

Efeito genético aditivo direto

Direct additive genetic effect

PN $(B W)$

GNDA $(W G)$

PD $(W W)$

Efeito genético aditivo materno

Maternal additive genetic effect

GNDA $(W G)$

0,15

Efeito de ambiente permanente

Permanent environmental effect

$\mathrm{PN}(B W)$

GNDA $(W G)$

PD $(W W)$

0,14

70,97

0,18

0,97

Efeito de ambiente temporário

Temporary environmental effect

PN $(B W)$

GNDA $(W G)$

PD $(W W)$

$-0,54$

0,96

315,65

Efeito fenotípico

Phenotypic effect

$\mathrm{PN}(B W)$

GNDA $(W G)$

$\mathrm{PD}(W W)$ resultados foram bastante semelhantes ao da análise univariada $(\mathrm{PN}=0,32$ e GNDA $=0,17)$, porém, na análise do PD com o GNDA, que resultou em herdabilidade de 0,32 para PD e 0,42 para GNDA, houve maior diferença em relação aos resultados da univariada $(\mathrm{PD}=0,12$ e $\mathrm{GNDA}=0,17)$.

As tendências genéticas diretas observadas (Figuras 1, 2 e 3 ) foram significativas para as três características, indicando tendência linear crescente do progresso genético ao longo dos anos para GNDA e PD (47 g/ano e $55 \mathrm{~g} /$ ano, respectivamente), enquanto, para o $\mathrm{PN}$, se manteve praticamente estável no período estudado ( $-2 \mathrm{~g} / \mathrm{ano})$. Os resultados para a tendência genética do PN são similares aos encontrados por Fernandes et al. (2002), que obtiveram leve declínio para o PN (-10_g/ano), mas não encontraram significância para essa tendência. Oliveira et al. (1995) e Ledic et al. (1988) verificaram tendência genética direta positiva para PN (13 g/ano e 24 g/ano), contudo, essa tendência foi muito baixa. Mello et al. (2002), por sua vez, constataram tendência de $46 \mathrm{~g}$ /ano para animais da raça Canchim, valor bem superior aos demais relatados. Os resultados para a tendência genética do GNDA (47 g/ano) confirmam a maioria dos reportados na literatura, como os de Fernandes et al. (2002), que obtiveram aumento anual de 40,85 g para bovinos Charolês, Euclides Filho et al. (1997), que obtiveram tendência direta de $67 \mathrm{~g} /$ ano para animais da raça Guzerá, e Euclides Filho et al. (2000), que estimaram tendência direta de $68,84 \mathrm{~g}$ /ano para população de animais Gir. A tendência genética do PD (55,1 g/ano) foi bem inferior à encontrada por Ferraz Filho et al. (2002), de 134 g/ano para bovinos Tabapuã, e por Mello et al. (2002), de 1,336 kg/ano para animais da raça Canchim.

Nas Figuras 4 e 5 são descritas as tendências genéticas maternas obtidas para GNDA e PD, ambas significativas ( $\mathrm{P}=0,001)$, com declínio ao longo do período, no mesmo valor de $-24 \mathrm{~g} /$ ano para GNDA e PD. Estas estimativas negativas devem-se à correlação negativa efeito direto $\mathrm{x}$

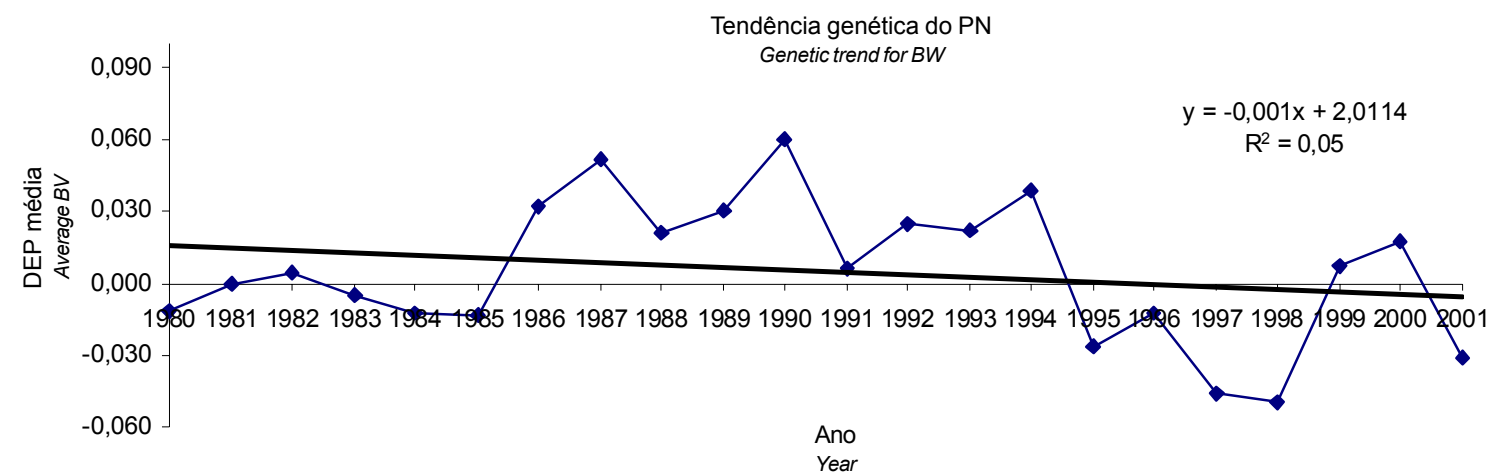

Figura 1 - Tendência genética do PN.

Figura 1 - Genetic trend for BW. 
efeito materno. Portanto, aumentando o efeito direto, diminui-se o materno. Para o GNDA, o resultado foi bem próximo ao encontrado por Fernandes et al. (2002), de -10 g/ano, porém bem abaixo do encontrado por Euclides Filho et al. (2000), que relataram tendência de $22 \mathrm{~g} /$ ano, Euclides Filho et al. (1997), de $24 \mathrm{~g} /$ ano, e Silva et al. (1997), de $226 \mathrm{~g}$ /ano. Entretanto, para PD, os resultados estão contrários aos obtidos por Mello et al. (2002), de $106 \mathrm{~g} /$ ano.

As tendências fenotípicas, ou seja, considerando as frações genética e não-genética da população, foram significativas para as características de PN (231 g/ano) e
GNDA (1.657 g/ano). Não foi incluída a tendência fenotípica do peso à desmama porque este peso não foi ajustado para idade-padrão e, portanto, as variações de base tem efeito muito grande sobre esta característica. Nas Figuras 6 e 7, é possível observar que o PN e o GNDA estão aumentando com o tempo. Para o PN, onde houve pequeno declínio genético e, fenotipicamente, houve aumento anual, provavelmente de origem ambiental. Para o GNDA, o progresso genético foi acompanhado do progresso fenotípico e, portanto, paralelo ao melhoramento genético, o ambiente foi favorável à expressão desta característica.

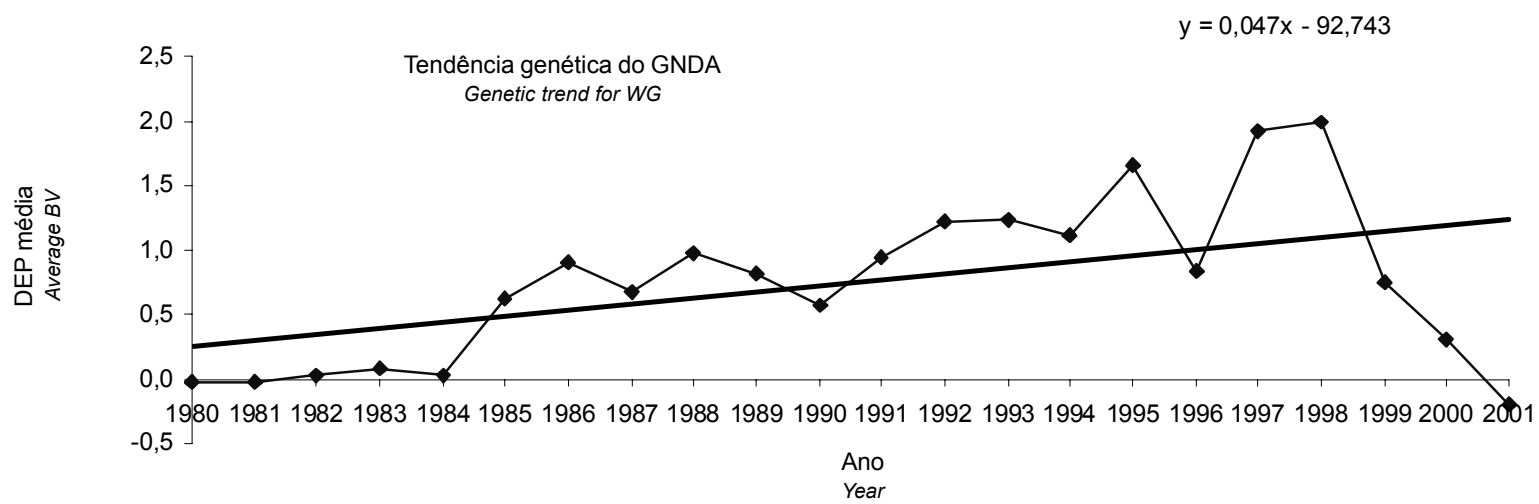

Figura 2 - Tendência genética do GNDA.

Figura 2 - Genetic trend for WG.

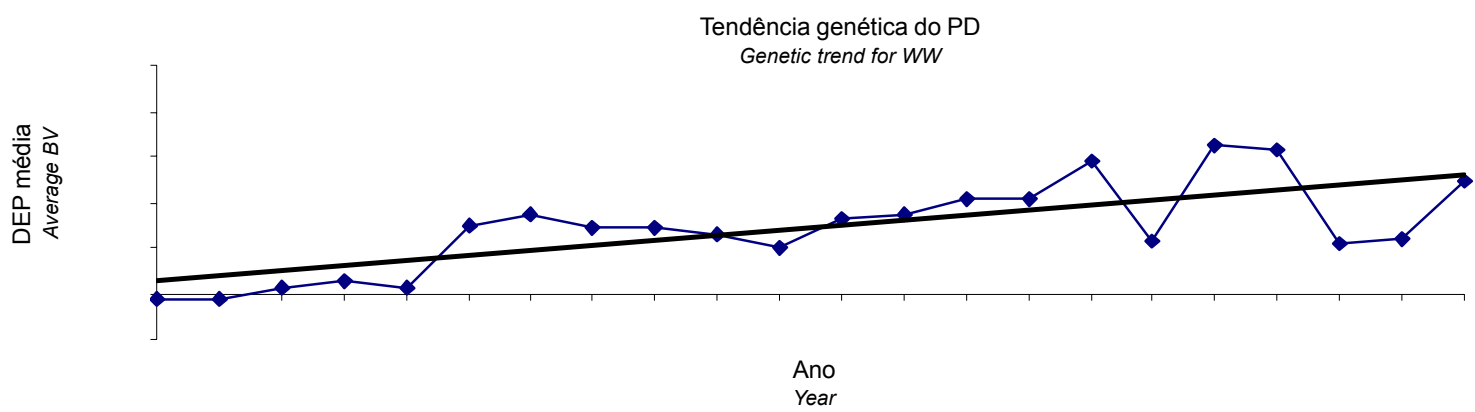

Figura 3 - Tendência genética do PD.

Figura 3 - Genetic trend for WW.

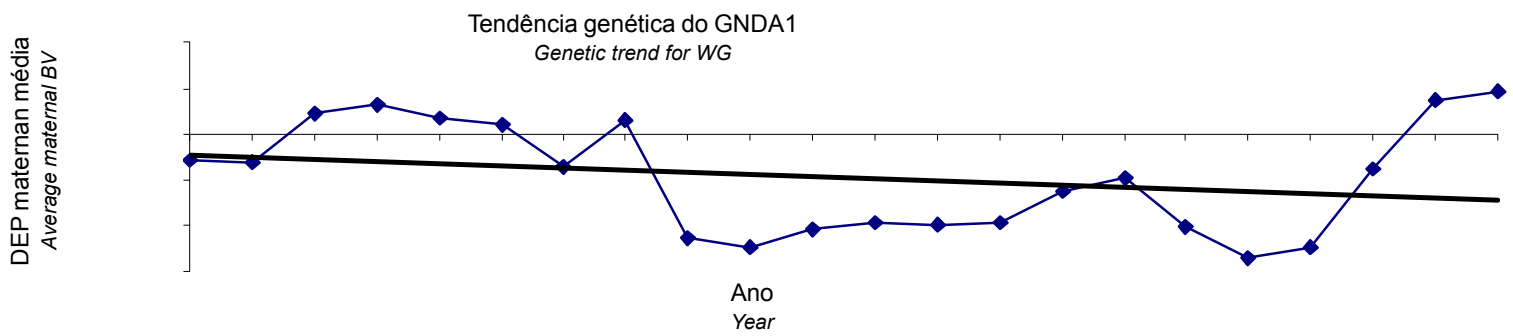

Figura 4 - Tendência genética materna do GNDA

Figura 4 - Maternal genetic trend for WG. 


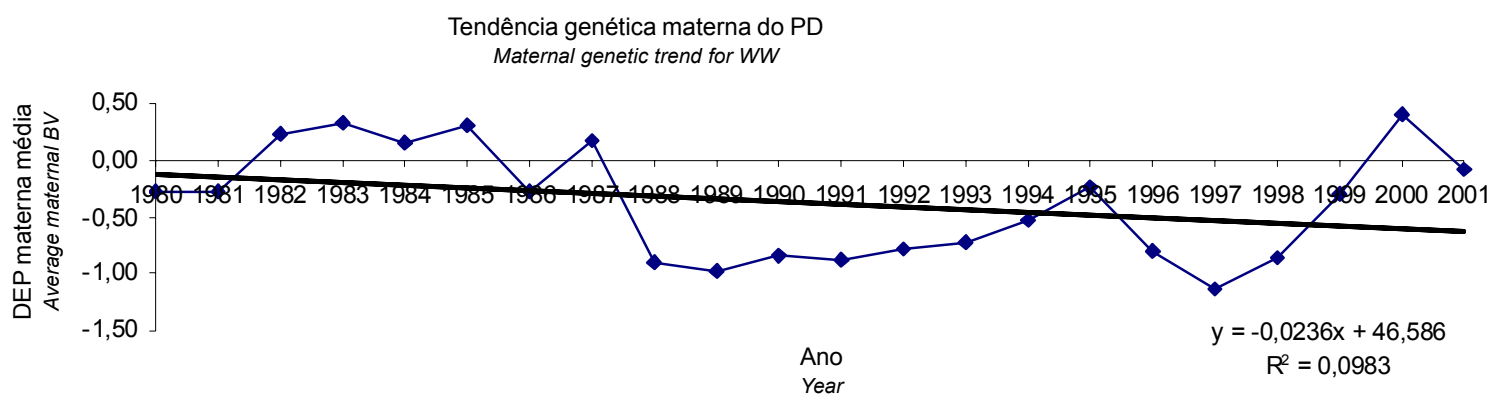

Figura 5 - Tendência genética materna do PD.

Figura 5 - Maternal genetic trend for WW.

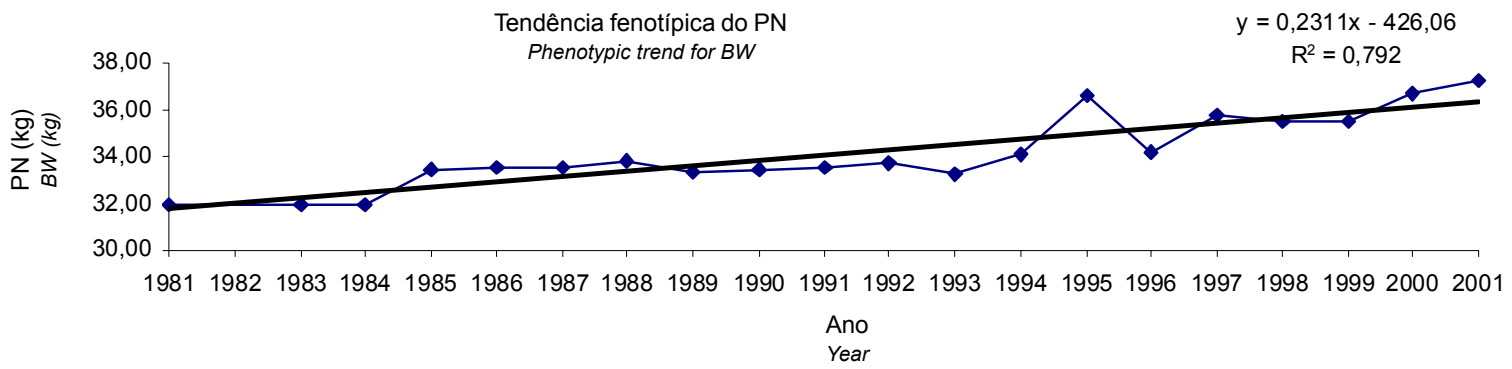

Figura 6 - Tendência fenotípica do PN.

Figura 6 - Phenotypic trend for BW.

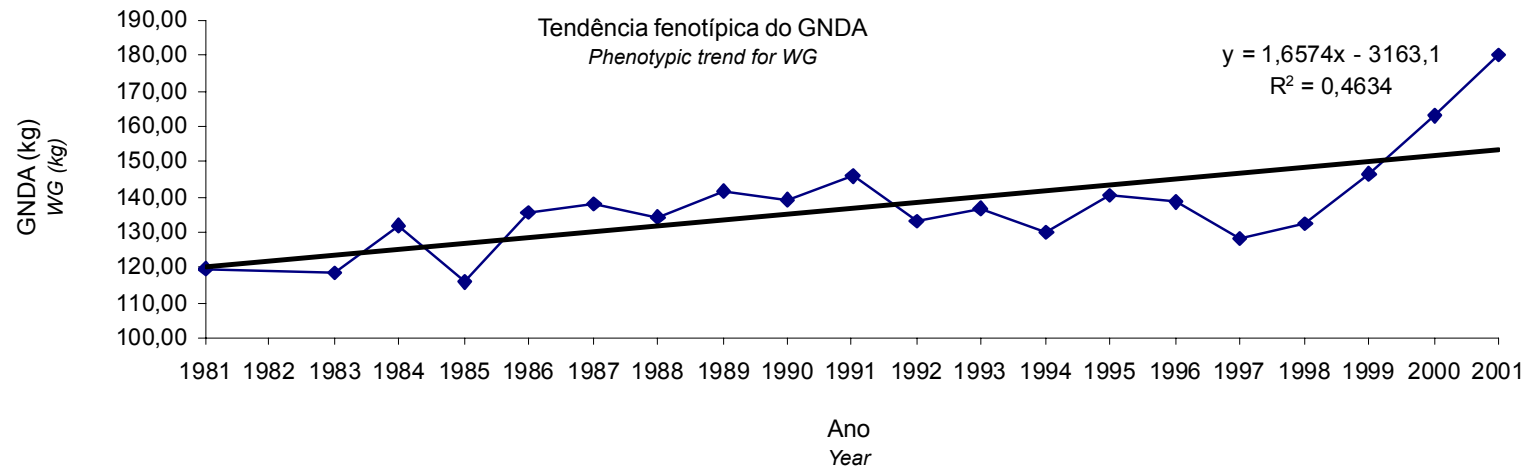

Figura 7 - Tendência fenotípica do GNDA.

Figura 7 - Phenotypic trend for WG.

\section{Conclusões}

As estimativas das herdabilidades genéticas médias para os pesos e ganhos de peso à desmama são importantes indicativos de que a seleção por estas características pode ser eficientemente para melhorar os rebanhos Devon no Rio Grande do Sul, assim como as correlações entre estas características, altas e positivas, denotam que a seleção pelo ganho de peso nesta fase, quando empregada nos rebanhos, pode acarretar resposta correlacionada satisfatória com o peso à desmama sem causar incremento no peso ao nascer, com o qual houve menor correlação.

Pela significância das tendências genéticas verificadas, pode-se inferir que, na população em questão, há um progresso genético nas características de ganho de peso do nascimento à desmama, enquanto, para o peso ao nascer, há um estádio de platô, o que é desejável para evitar problemas de parto. 


\section{Literatura Citada}

BIFFANI, S.; MARTINS FILHO, R.; GIORGETTI, A. et al. Fatores ambientais e genéticos sobre o crescimento ao ano e ao sobreano de bovinos Nelore, criados no nordeste do Brasil. Revista Brasileira de Zootecnia, v.28, n.3, p.468-473, 1999.

BOLDMAN, K.G.; KRIESE, L.A.; Van VLECK, L.D. et al. A manual for use of MTDFREML. Lincoln: U.S. Department Agriculture, Agricultural Research Service, 1995. 120p.

BOLIGON, A.A.; WEBER, T.; KIPPERT, C.J. et al. Componentes de variância e parâmetros genéticos para características de crescimento para a raça Charolês no estado do Rio Grande do Sul. In: REUNIÃO ANUAL DA SOCIEDADE BRASILEIRA DE ZOOTECNIA, 41., 2004, Campo Grande. Anais... Campo Grande: V2 Comunicação, 2004 (CD-ROM). Melhoramento Animal. MELH 015.

CARDELLINO, R.A.; OSÓRIO, J.C.S. Melhoramento animal para Agronomia, Veterinária e Zootecnia. Pelotas: Editora Universitária, 1998. 153p.

CARDELlino, R.A.; ROVIRA, J. Mejoramiento genetico animal. Montevideo: Editora Agropecuária Hemisfério Sur, 1987. $253 \mathrm{p}$

CARDOSO, F.F. Caracterização genética do desempenho do nascimento à desmama de bovinos Aberdeen Angus criados no Rio Grande do Sul. Pelotas: Universidade Federal de Pelotas, 1999. 116p. Dissertação (Mestrado em Ciências) Universidade Federal de Pelotas, 1999.

CARDOSO, F.F.; CARDELlinO, R.A.; CAMPOS, L.T. Fatores ambientais que afetam o desempenho de nascimento à desmama de bezerros Angus criados no Rio Grande do Sul. Revista Brasileira de Zootecnia, v.30, n.2, p.326-335, 2001.

DAL-FARRA, R.A.; ROSO, V.M.; SCHENKEL, F.S. Efeitos de ambiente e de heterose sobre o ganho de peso do nascimento ao desmame e sobre os escores visuais ao desmame de bovinos de corte. Revista Brasileira de Zootecnia, v.31, n.3, p.13501361, 2002 (supl.).

ELER, J.P.; Van VLECK, L.D.; FERRAZ, J.B. et al. Estimation of variances due to direct and maternal effects for growth traits of Nelore cattle. Journal of Animal Science, v.73, p.3253-3258, 1995 .

EUCLIDES FILHO, K.; SILVA, L.O.C.; ALVES, R.G.O. et al. Tendências genéticas na raça Indubrasil. In: REUNIÃO ANUAL DA SOCIEDADE BRASILEIRA DE ZOOTECNIA, 34., 1997, Juiz de Fora. Anais... Juiz de Fora: Sociedade Brasileira de Zootecnia, 1997. p.171-172.

EUCLIDES FILHO, K.; SILVA, L.O.C.; ALVES, R.G.O. et al. Tendências genéticas na raça Gir. Pesquisa Agropecuária Brasileira, v.35, n.4, p.787-791, 2000.

FERNANDES, H.D.; FERREIRA, G.B.B.; RORATO, P.R.N. Tendências e parâmetros genéticos para características prédesmama em bovinos da raça Charolês criados no Rio Grande do Sul. Revista Brasileira de Zootecnia, v.31, n.1, p.321-330, 2002 (supl.).

FERRAZ FILHO, P.B.; RAMOS, A.A.; SILVA, L.O.C. et al. Tendência genética dos efeitos direto e materno sobre os pesos à desmama e pós-desmama de bovinos da raça Tabapuã no Brasil. Revista Brasileira de Zootecnia, v.31, n.2, p.635-640, 2002.

$\mathrm{KOCH}, \mathrm{M} . \mathrm{R}$. The role of material effect in animal breeding. IV. Maternal effects in beef cattle. Journal of Animal Science, v.35, p.1316-1323, 1972.

KOOTS, K.R.; GIBSON, J.P.; WILTON, J.W. Analysis of published genetic parameters estimates for beef production traits. 1 - Herdability. Animal Breeding Abstract, v.62, n.5, p.309-338, 1994.
LAUREANO, M.M.M.; FORNI, S.; COSTA, R.B. et al. Estimativas de parâmetros genéticos para características de crescimento do nascimento à desmama em animais da raça Nelore " 1 ". In: REUNIÃO ANUAL DA SOCIEDADE BRASILEIRA DE ZooteCniA, 41., 2004, Campo Grande. Anais... Campo Grande: V2 Comunicação, 2004 (CD-ROM). Melhoramento Animal. MELH 152.

LEDIC, I.L.; NOBRE, P.R.C.; ROSA, A.N. Tendências fenotípicas, ambientais e genéticas estimadas para os pesos ao nascer (PN), aos 205 (P205), 365 (P365) e 550 (P550) dias de idade de animais da raça Tabapuã. In: REUNIÃO ANUAL DA SOCIEDADE BRASILEIRA DE ZOOTECNIA, 25., 1988 , Viçosa, MG. Anais... Viçosa, MG: Sociedade Brasileira de Zootecnia, 1988. p. 238.

LIMA, A.E.S.; FERRAZ FILHO, P.B.; SILVA, L.O.C. et al. Efeitos genéticos e ambientais em pesos a desmama de bovinos da raça Nelore mocha, em diferentes regiões pecuárias do estado de Goiás, Brasil In: REUNIÃO ANUAL DA SOCIEDADE BRASILEIRA DE ZOOTECNIA, 41., 2004, Campo Grande. Anais... Campo Grande: V2 Comunicação, 2004 (CD-ROM). Melhoramento Animal. MELH 008.

LOBO, R.B. Programa de melhoramento genético da raça Nelore. Ribeirão Preto: Universidade de São Paulo, 1994. 54p.

LOBO, R.N.B.; MADALENA, F.E.; DE LOS REYES, A. Average estimates of genetic parameters for beef an dairy cattle in tropical regions. Animal Breeding Abstract, v.68, p.433$462,2000$.

MARTINS, G.A.; FILHO, R.M.; LIMA, F.A.M. et al. Influência de fatores genéticos e de meio sobre o crescimento de bovinos da raça Nelore no estado do Maranhão. Revista Brasileira de Zootecnia, v.29, n.1, p.103-107, 2000.

MELLO, S.P.; ALENCAR, M.M.; SILVA, L.O.C. et al. Estimativas de (Co)variâncias e tendencies genéticas para pesos em um rebanho Canchim. Revista Brasileira de Zootecnia, v.31, n.4, p.1707-1714, 2002.

MERCADANTE, M.E.Z., LOBO, R.B. Estimativas de (co)variâncias e parâmetros genéticos dos efeitos direto e materno de características de crescimento de fêmeas de um rebanho Nelore. Revista Brasileira de Zootecnia, v.26, n.6, p.1124-1133, 1997.

OLIVEIRA, J.A.; LÔBO, R.B.; OLIVEIRA, H.N. Tendência genética em pesos e ganhos em peso de bovines da raça Guzerá. Pesquisa Agropecuária Brasileira, v.30, n.11, p.1355-1360, 1995.

STATISTICAL ANALYSES SYSTEM - SAS. SAS/STAT. User's guide. 6.11.ed. Cary: 1996.

SILVA, I.S.; PACKER, I.U.; SILVA, L.O.C. et al. Parâmetros genéticos para características de crescimento de bovinos da raça Guzerá usando análises unicaracterísticas e bicaracterísticas (1) In: REUNIÃO ANUAL DA SOCIEDADE BRASILEIRA DE ZOOTECNIA, 41., 2004, Campo Grande. Anais... Campo Grande: V2 Comunicação, 2004 (CD-ROM). Melhoramento Animal. MELH 080.

WILLHAM, R.L. The role of maternal effect in animal breeding: III. Biometrical aspects of maternal effects in animals. Journal of Animal Science, v.35, p.1288-1302, 1972. 